Voix et Images

voixetimages

\title{
Un magnifique voyage dans le temps
}

\section{Lucie Joubert}

Volume 29, numéro 2 (86), hiver 2004

Le laboratoire de l'écriture : manuscrits et variantes

URI : https://id.erudit.org/iderudit/008782ar

DOI : https://doi.org/10.7202/008782ar

Aller au sommaire du numéro

\section{Éditeur(s)}

Université du Québec à Montréal

\section{ISSN}

0318-9201 (imprimé)

1705-933X (numérique)

Découvrir la revue

\section{Citer cet article}

Joubert, L. (2004). Un magnifique voyage dans le temps. Voix et Images, 29(2), 179-183. https://doi.org/10.7202/008782ar d'utilisation que vous pouvez consulter en ligne.

https://apropos.erudit.org/fr/usagers/politique-dutilisation/ 


\title{
F É M I N I S M E S
}

Un magnifique voyage dans le temps

$+++$

\author{
LUCIE JOUBERT \\ Université d'Ottawa
}

Commençons par une absence, histoire de vider la question: dans les 750 pages bien comptées de cette anthologie ${ }^{1}$ par ailleurs magnifique, aucune, AUCUNE, n'est consacrée aux textes féministes littéraires. Silence total sur la réflexion de Suzanne Lamy dans D'elles et Quand je lis, je m'invente; silence tout aussi incompréhensible autour d'œuvres fondatrices, toutes publiées avant 1985, comme L'Euguélionne de Louky Bersianik (la reproduction de la couverture en page 402 exceptée), Lueur de Madeleine Gagnon ou L'Amèr ou le chapitre effrité de Nicole Brossard, qui clamait pourtant «écrire: je suis une femme est plein de conséquences». Au Québec, les littéraires ont souvent été les laissées-pour-compte des événements féministes: leurs ouvrages ne font pas le poids à côté des statistiques des sociologues, des faits des journalistes, des dates des historiennes. Les colloques, les débats internationaux, les publications sur le féminisme, les politiques éditoriales des maisons d'édition nous le rappellent régulièrement. Rarement, toutefois, aurons-nous eu une preuve aussi éclatante du peu de cas qu'on fait de l'imaginaire des femmes et du rôle qu'il a joué et qu'il joue toujours dans l'évolution de cette pensée féministe dont on l'évince: création de personnages féminins forts, qui rompent avec les traditions; appropriation de lieux et d'espaces jadis réservés aux hommes; narration privilégiant la perspective féminine; recherche d'une langue autre, volonté de questionner les genres, et plus encore.

On prétend, à la page 25, que cette élimination vient de la noble intention de ne pas «charcuter les écrits»: à d'autres. Il aurait été très simple de glisser, à même les différentes thématiques abordées, des extraits littéraires qui accompagnent le sujet, ou tout bonnement de créer une catégorie à part: les écrivaines n'en sont pas à une marge près. En invoquant l'impossibilité de «réduire» des textes comme Les fées ont soif de Denise Boucher «à quelques pages» (25), les auteures font mine de placer le texte littéraire dans une catégorie supérieure, intouchable: pirouette habile pour mieux se débarrasser du problème. On alléguera enfin la présence des textes de Françoise Loranger, Gloria Escomel, Laure Conan, Eva Circé-Côté et, ultime ruse, de Lucile Durand, alias Louky Bersianik: c'est oublier qu'elles figurent dans l'anthologie en tant que signataires

$$
+++
$$

1 Micheline Dumont et Louise Toupin (dir.), La pensée féministe au Québec. Anthologie [1900-1985], Montréal, Les éditions du remueménage, 2003, $750 \mathrm{p}$. 
de textes argumentatifs et non de fiction. On dit souvent que les progrès sont l'œuvre de visionnaires; décidément, en matière de condition féminine, le mot est à prendre ici dans son sens le plus étroit: la vision, on veut bien, dans la mesure où elle reste assujettie à la réalité. Bel oxymoron, feraient remarquer les littéraires, si on leur en donnait la chance...

Ce préambule ayant permis de mettre en relief la lacune de cet ouvrage et ayant servi d'exutoire à une certaine frustration que je sais être partagée par plusieurs littéraires, force est d'admirer le travail monumental qu'ont accompli Dumont et Toupin. Divisé en trois parties principales, l'ouvrage présente d'abord «le féminisme et les droits des femmes (1900-1945)», «le féminisme comme groupe de pression (1945-1985)» et "le féminisme comme pensée radicale (1969-1985)», le chevauchement des deux dernières parties étant justifié par la coexistence de deux courants distincts, la voie égalitaire et la voie radicale.

De la première partie se dégagent trois impressions principales: actualité, ouverture et fluidité rhétorique. Mis à part l'empressement des signataires à indiquer que les revendications et les gains subséquents putatifs ne les empêcheront pas de continuer à être de bonnes épouses et de bonnes catholiques (que de longs gants blancs il fallait mettre à l'époque pour avoir le privilège de s'exprimer), les arguments, les exposés, les faits demeurent singulièrement familiers. "Payez-vous le luxe d'une trâlée de mioches, écrit Éva Circé-Côté en 1920, quand il existe une entente tacite entre les propriétaires pour ne pas louer leur maison à des gens qui ont des enfants!» (203) : comment ne pas penser à la crise du logement qui affecte les grandes villes et aux discriminations qu'elle entraîne inévitablement? Marie-Louise Brodeur, en 1915, y va d'un plaidoyer qui rappelle la thèse de Naomi Klein dans No Logo:

\footnotetext{
Acheter est un acte social et pas seulement économique. Mais bien peu de femmes du monde en ont conscience. [...] Car si [une] personne favorisée de la fortune se demandait un seul instant à quel salaire de famine ont été réduites les ouvrières qui confectionnent ces objets qui permettent aux marchands de vendre à des prix dérisoires des effets de lingeries et de mode dont le profit est pris sur le travail et l'activité angoissée de l'ouvrière [...], sûrement elle éviterait aux employées l'existence excessivement pénible qu'elle leur impose. (85)
}

Cette vision sociale, qui rejoint à bien des égards les préoccupations contemporaines, subsume par sa fougue et sa verdeur ce qui pourrait apparaître comme une naïveté devant les enjeux d'une économie industrielle qui commence à faire ses ravages.

Toutes ces femmes qui rédigent article sur article, prennent la parole à l'occasion de conférences, fouettent le moral des troupes pour ainsi dire, étayent leurs propos par des lectures et des recherches approfondies qui les relient au reste du monde et cautionnent leurs positions: "Au moyen-âge, argue Marie-Justine Gérin-Lajoie, les femmes étaient admises dans les corporations ouvrières sur un pied d'égalité avec les hommes. Elles y avaient droit aux mêmes dignités, aux mêmes honneurs» (96). Et de citer des sources pour parer aux objections. Idola Saint-Jean, de son côté, reprend la définition du féminisme que donna Léontine Zanta, la première femme à recevoir un doctorat en philosophie de l'Université de Paris: «Le féminisme n'est pas une rêverie d'utopiste, une boutade de cerveaux exaltés, c'est la revendication juste et légitime de la femme à ses droits d'être humain» (57). Inspirées, donc, par les avancées et les luttes de leurs consœurs européennes, les féministes d'ici s'inscrivent dans un mouvement de 
revendication qui puise une partie de sa force dans la connaissance de ce qui se passe ailleurs. Une telle volonté de s'insérer dans la société, de briser l'isolement qui est le lot des femmes, se traduit symboliquement par la recommandation d'Édith Méthot aux fermières en 1931: "Que la fermière qui ne peut se payer du luxe sans péril se procure un radio, merveilleux véhicule de distractions instructives et éducatrices, venant de toutes parts» (104). Détail qui n'en est pas un: la radio, on le redécouvre à chaque panne d'électricité, est la dernière ressource quand le reste du monde semble nous échapper...

Tant d'information à la fois aurait pu mener à un nivellement du discours, du point de vue stylistique, s'entend; il n'en est rien. Par la façon judicieuse d'alterner les textes journalistiques, les mémoires, les conférences publiques, les requêtes et les rapports de toutes sortes, l'anthologie permet la démonstration de différents styles d'écritures et de contextes: les journalistes, dont la très (trop?) présente Éva Circé-Côté, proposent des articles plus résolument primesautiers, compte tenu du lectorat, et allègent les débats de fond par un regard distancié et l'humour qui l'accompagne:

\footnotetext{
$\mathrm{M}^{\mathrm{Ile}}$ Jeanne Beneben (étudiante en France), après un examen très sérieux, note Robertine Barry dans La Patrie, avait été admise à la licence en droit, et [est] sortie bonne première d'un concours où tous les autres compétiteurs portaient barbiche. [...] Que dire de cette pensée d'Aristote, qu'on lit dans une de ses œuvres: «Les Mityléniens honorèrent Sapho, quoique ce fut une femme». Cela ne donne-t-il pas la mesure du préjugé barbifère? (63-65)
}

Façon élégante de mettre à mal, en même temps, Molière et son célèbre «du côté de la barbe est la toute-puissance», un Molière souvent pris à partie (preuve que la littérature sert à quelque chose) comme en fait foi le texte «Les femmes savantes» de Joséphine Marchand-Dandurand, paru en 1895: «Si la terreur [des hommes] de se voir égalés ou surpassés les inspirent, qu'ils nous permettent encore une fois de calmer leurs alarmes. Nous sommes si éloignées de leur porter ombrage que quand nous parlons d'étudier ou de cultiver la littérature, nous n'entendons que dissiper un peu les voiles de notre profonde ignorance» (62). Époque bénie, ai-je envie d'insinuer, où les hommes croyaient encore les femmes incapables d'ironie!

On sera séduit aussi par la rigueur rhétorique de Marie Gérin-Lajoie, qui force l'admiration par des exposés limpides sur les enjeux réels du droit de vote des femmes, par l'empathie sincère d'Idola Saint-Jean ou de Thérèse Casgrain, cette dernière que l'on ne peut s'imaginer qu'en manteau de fourrure, bijoux à l'avenant:

\footnotetext{
J'aborde un sujet qui, je le sais, déplaît à plusieurs. Tant pis, ce qui me pousse à le traiter est trop important, trop vital pour que des considérations accessoires, même respectables, puissent empêcher qu'on dise ce qui doit en être dit. Pour couper court aux préambules et précautions, je pose une question à plusieurs branches: Pourquoi l'institutrice primaire catholique est-elle payée à peu près le dixième de ce qu'est payée l'institutrice ontarienne? Pourquoi les trois quarts des jeunes filles qui s'essaient à ce métier l'abandonnent-elles à la première occasion? Pourquoi l'existence d'un bureau central qui accorde des diplômes d'enseignement à des enfants qui viennent de terminer leur sixième année primaire? [...] Pourquoi, enfin, parler en termes émus de l'école du rang, de la nécessité de ruraliser l'école de la campagne, de l'urgence de remonter le niveau moyen de l'instruction, du devoir qui incombe aux pédagogues et de lire et de se renseigner, quand on persiste à traiter la femme qui enseigne comme on ne traite pas une apprentie d'usine? (107-108)
} 
Toutes ces femmes lettrées, scolarisées, qui ont subi, imperturbables et déterminées, les quolibets des foules (ceux des femmes aussi, souvent) mais ont persisté à revendiquer, nous sont, en quelque sorte, rendues par l'anthologie. Elles redeviennent, par ce retour vers le passé, des complices, des sœurs de luttes, comme des parentes éloignées dont on redécouvre l'existence après des années d'absence.

Sur le plan formel, l'ouvrage non seulement donne accès à des textes introuvables pour quelques-uns mais replace ces mêmes textes dans leur période de diffusion en ajoutant des points de repère nombreux et précis pour retracer, en quelques phrases, les faits saillants biographiques ou événements marquants d'une société en effervescence. Les paragraphes de présentation attirent habilement l'attention sur des anecdotes entourant la rédaction ou la parution du texte ou sur un détail qui pourrait échapper au lecteur pressé. Ainsi, nous font remarquer les auteures, Éva Circé-Côté encore une fois, emportée par son propos dans "La grève des femmes», en oublie jusqu'à son pseudonyme de Julien Saint-Michel et «écrit nous les femmes avec conviction» (210)!

Plus loin, en introduction au collage de textes de Monique Béchard (1948-1952) qui se porte à la défense du cours classique pour les filles, les auteures racontent:

\footnotetext{
M ${ }^{\mathrm{gr}}$ Albert Tessier, responsable et promoteur des écoles ménagères, critiquait les «béchardises». Il l'accuse de raisonner comme Simone de Beauvoir, c'est-à-dire mal. Enrobée dans les théories de psychologie différentielle, la thèse de Monique Béchard met en évidence les anomalies des idées reçues. Elle a l'audace de critiquer Gina Lombroso, dont les écrits servent de Bible aux ennemis de l'instruction des filles. (232)
}

Efficaces, ces amorces ont le grand mérite de synthétiser le propos à venir et de fournir des pistes supplémentaires à qui voudrait approfondir la question, sans pour autant briser le rythme soigneusement entretenu par des articles d'à peu près égale venue.

Les deux autres parties de l'anthologie réserveront peut-être moins de surprises à quiconque s'est un tant soit peu intéressé aux questions reliées à la condition féminine contemporaine; les visages, les voix ou les textes des actrices de ces périodes nous sont encore familiers et présents à l'esprit: Lise Payette, Simonne Monet-Chartrand, Léa Roback, Armande Saint-Jean et bien d'autres. Encore que... Il est émouvant d'entendre l'appel à la solidarité lancé par Mary Two-Axe Early qui explique en 1968 l'ultime «deux poids, deux mesures» dont sont victimes les Amérindiennes:

\footnotetext{
Les Indiens du Canada ont droit à certains avantages pour leur éducation au niveau primaire, secondaire et universitaire. La femme indienne qui épouse un non-Indien perd ces privilèges, ce qui l'empêche souvent de poursuivre les études supérieures commencées lorsqu'elle était célibataire et considérée comme un membre de la bande. Les enfants des Indiennes mariées à des non-Indiens qui vivent sur notre réserve doivent voyager des milles par autobus matin et soir pour se rendre dans des écoles où les élèves se montrent hostiles à la présence de demiIndiens qui envahissent leur domaine. Les femmes non indiennes mariées à des membres de la bande et leurs enfants ont droit à tous les avantages à tous les niveaux d'éducation. En outre, leurs enfants ne sont pas envoyés par autobus dans des écoles éloignées. (349)
}

Effet de la traduction maison, peut-être, ce texte, qui commence humblement par «Chère Madame Bird», se lit comme une complainte et non comme un cri, allant au plus pressé pour exposer l'injustifiable. 
Il est bon aussi (et triste en même temps) de se rappeler que Châtelaine a déjà été féministe, n'hésitant pas à publier en 1964, par exemple, un article de Fernande SaintMartin intitulé «Un mythe à détruire: aucun matriarcat au Québec» (338), dont le titre seul fait mesurer le gouffre qui sépare la revue actuelle de celle qu'elle fut. Il est passionnant, enfin, de voir défiler à rebours, comme un film que l'on rembobine, certains événements qui ont balisé la route vers l'émancipation: création du RAIF, du Front de libération des femmes et parution de son brûlot Québécoises deboutte! (la société avait encore l'épiderme assez sensible à l'époque); métamorphose de la revue La Vie en rose qui passait du feuillet inséré dans Le Temps fou à une existence de grande fille, si l'on peut dire, ainsi que certains débats et cas de conscience qui ont occupé les réunions de la rédaction, dont celui relié à la publication du centerfold érotique de Nicole Morisset. On lit, à cet égard:

Ça n'a pas été facile. On ne devient pas une revue cochonne du jour au lendemain. D'ailleurs, beaucoup de ce que nous annoncions dans notre célèbre teaser (Le Temps fou, n 8) - le désir d'outrepasser, de déborder, d'enjamber les ghettos, d'appeler à l'imaginaire, d'être excessives - sont des choses plus faciles à dire qu'à réaliser, il a bien fallu s'en rendre compte. En ce sens, on peut dire que nous avons vécu notre premier grand test au sujet de l'érotisme. Nous ne l'avions pas vu venir, celui-là, et nous nous retrouvions tout à fait démunies devant une question encore tabou parmi nous. Le cul nous a fait peur. (583)

Verdeur du langage, nouvelle liberté; les articles plus récents affichent une souplesse linguistique, pas toujours heureuse, hélas: certains textes (des Têtes de pioche entre autres), dans leur envie de rejoindre le prolétariat peut-être, font regretter par leur populisme - qui l'eût cru! - la rhétorique austère de leurs aînées.

Mais toutes ces paroles, de tous horizons, font de l'anthologie une somme dorénavant obligatoire dans toute bibliothèque, féministe ou pas. L'ouvrage se clôt d'ailleurs sur... une ouverture, autre oxymoron qui illustre bien la synergie dégagée par tous ces textes que l'on a peine à quitter: ouverture de la société québécoise aux revendications des lesbiennes, des immigrantes, des marginalisées dans une volonté de solidarité; aperçu aussi de ce que le Québec est en train de devenir depuis 1985. Dumont et Toupin terminent ainsi leur traversée du temps: «En 1900, comme en 1985 et comme en 2000, cette perspective unique sur le monde qu'est le féminisme ne laisse rien intact après son passage. Cette onde de choc ravive la démocratie, appelle l'égalité et la liberté.» (730)

C'est sans doute parce que le tableau ainsi recréé des luttes des femmes est si vibrant, si plein de promesses, d'enthousiasme et de courage, malgré les défaites, les reculs et les inquiétudes, que j'aurais tant aimé que la littérature y figure; subsiste à la fin de la lecture, mêlée au plaisir de tant de découvertes, la mélancolie de ne pas avoir rencontré de littéraires à ce party de filles. Aux romancières, poètes, dramaturges, essayistes et nouvellières, maintenant, de faire leur bilan. 